\title{
CLINICAL COURSE OF ACANTHAMOEBA KERATITIS BY GENOTYPES T4 AND T8 IN HUNGARY
}

\author{
ERIKA OROSZ ${ }^{1 *}$, DOROTTYA KRISKÓ ${ }^{2}$, LEI SHI ${ }^{3}$, GÁBOR L. SÁNDOR ${ }^{2}$, \\ HubA J. KISS ${ }^{2}$, BERTHOLd SEITZ ${ }^{3}$, ZOLTÁN ZSOLt NAGY ${ }^{2}$ \\ and NÓRA SZENTMÁRY ${ }^{2,3}$ \\ ${ }^{1}$ Department of Parasitology, National Public Health Center, Budapest, Hungary \\ ${ }^{2}$ Department of Ophthalmology, Semmelweis University, Budapest, Hungary \\ ${ }^{3}$ Department of Ophthalmology, Saarland University Medical Center, \\ Homburg, Germany
}

(Received: 5 December 2018; accepted: 17 January 2019)

Genus Acanthamoeba is an opportunistic protozoan that is widely distributed in the environment. Within this genus, numerous species are recognized as human pathogens, potentially causing Acanthamoeba keratitis (AK). AK is a corneal disease, associated predominantly with contact lens (CL) wear; its epidemiology is related to the specific Acanthamoeba genotypes. This study reports seven CL wearer, Acanthamoeba PCR-positive patients with AK, diagnosed between January 2015 and 2018. Patients had the diagnosis of AK 1.36 months after first symptoms. Genotyping allowed the identification of six isolates of the T4 and one of the T8 genotypes. At first presentation, pseudendritiformic epithelopathy/dirty epithelium (four eyes, 57.1\%), multifocal stromal infiltrates (five eyes, $71.4 \%$ ), ring infiltrate (three eyes, $42.8 \%$ ), and perineuritis (one eye, $14.3 \%$ ) were observed. AK was healed without later recurrence in two eyes $(28.5 \%)$ using triple-topical therapy, in three eyes $(42.8 \%)$ following additional penetrating keratoplasty. In one patient (14.3\%), AK recurred following successful application of triple-therapy and was treated successfully with repeated triple-topical therapy and in one patient (14.3\%), no follow-up data were available after diagnosis. We could not observe correlation of genotype and clinical course or the necessity of corneal transplantation in our case series.

Keywords: Acanthamoeba keratitis, real-time FRET PCR, sequence analysis, clinical course

*Corresponding author; E-mail: orosz.erika@oki.antsz.hu 


\section{Introduction}

Acanthamoeba is a genus of free-living amebae widely distributed in various ecological environments. The spectrum ranges from natural biotopes, such as soil, plants, air, dust, freshwater, fishes, sea water, drinking water, swimming pools, and contact lenses (CLs). In addition, they have been isolated from animals such as fish and mammals and also from humans [1-3].

The first cases, which clearly established Acanthamoeba as a causative agent of disease in humans, were published in the early 1970s [4]. The pathogenesis of AK in humans is currently studied and different Acanthamoeba genotypes have been reported from all over the world [5].

The traditional Acanthamoeba classification has used morphological characteristics, such as morphology, size, and shape [6]. Modern classification uses a molecular biological approach based on $18 \mathrm{~S}$ ribosomal RNA (rRNA) gene to classify Acanthamoeba isolates as one of the 20 known genotypes (T1-T20). Detection of Acanthamoeba can be rapidly achieved using real-time molecular methods. Each genotype exhibits $5 \%$ or more sequence divergences between different genotypes [7]. For diagnostic purposes, the detection of Acanthamoeba at the genus level is sufficient to recognize whether an individual is infected or not. Acanthamoeba keratitis (AK) is mainly present in CL wearers, especially in case of prolonged use of CLs, unappropriate hygienic conditions (contact of the lenses with tap water, swimming pool, dust, etc.) or in case of corneal trauma. AK is mainly caused by isolates with T4 genotype [8-10]; however, T2, T3, T5, T6, T8, T9, T11, T13, and T15 genotype species have also been identified in patients with AK, as shown in Table I [11-18].

Following an appropriate clinical and also laboratory diagnosis (confocal microscopy, polymerase chain reaction, histology, and microbiological culture) and having Acanthamoeba in culture, it is still not possible to arrive at a conclusion in which topical treatment could be effectively used.

Table I. Non-T4 genotype Acanthamoeba in different countries (literature data)

\begin{tabular}{ll}
\hline Acanthamoeba genotypes & \multicolumn{1}{c}{ Report countries } \\
\hline T2 & Iran [12] \\
T3 & Iran [12], England [12], Austria, USA, Spain [15, 18], and Mexico [17] \\
T5 & Austria [16] and USA [14] \\
T6 & Austria [16] \\
T8 & Hungary [26] \\
T9 & Thailand [3] \\
T10 & Austria [16] \\
T11 & Austria [16], USA [11], and Spain [15] \\
T15 & Italy [13] \\
\hline
\end{tabular}


Our aim was to analyze the effect of Acanthamoeba genotype, isolated from human corneal scrapings and fluid from CL storage, on clinical course of AK.

\section{Materials and Methods}

We retrospectively collected diagnostic and clinical data of patients with $\mathrm{AK}$ [polymerase chain reaction (PCR) and/or culture positive], with AK diagnosis between January 2015 and 2018 at the Department of Ophthalmology of Semmelweis University.

\section{Sample collection}

Acanthamoeba was isolated from corneal scrapings of seven patients [three males (43\%) and four females (57\%), mean age during diagnosis 30.71 years] between January 2015 and 2018.

\section{Culture-confirmed detection method}

The seven samples of corneal scrapings were then transferred to Page's agar plates overlaid with heat-killed Escherichia coli and cultured at $37^{\circ} \mathrm{C}$ for 10 days. The morphology of trophozoites and cysts was studied by light microscopy, according to Page [19]. Plates were monitored for growth of ameba microscopically, from 72 to $96 \mathrm{~h}$ for the presence of Acanthamoeba spp. cysts and trophozoites under $320 \times$ and $400 \times$ magnification.

\section{Molecular methods}

Acanthamoeba was isolated from corneal scrapings of the patients and from fluid of CL storage case. The Acanthamoeba species were isolated by dilution method. For this purpose, the samples of corneal scrapings were suspended in $400-\mu$ physiological saline solution $(0.85 \%)$. After preparation, the DNA extraction was treated with High Pure PCR Template Preparation Kit (Roche, Germany) according to the manufacturer's instructions. If further processing was delayed, the isolates were stored at $4{ }^{\circ} \mathrm{C}$ for $24 \mathrm{~h}$ or at $-20{ }^{\circ} \mathrm{C}$ for a longer period. The DNA amplification was performed using genus-specific primers and genus-specific fluorescence resonance energy transfer (FRET) hybridization probes, previously described by Orosz et al. [20]. Each experiment included one reaction mixture 
without DNA as a negative control; positive control and each specimen was run in duplicate for real-time PCR assay in parallel.

PCR products were purified with PCR Clean-Up M Kit (Viogene, Sunville, $\mathrm{CA})$. The sequence of each amplicon was determined by cycle sequencing with primers for the 5'-NTR region and with primers with BigDye Terminator v3.1 Cycle Sequencing Kit (Applied Biosystems, Germany) according to the manufacturer's instruction. The electrophoresis was carried out using Applied Biosystems 3500 Genetic Analyzer.

The 5'-NTR and VP1 gene sequences were subject to nucleotide-nucleotide BLAST analysis [21] using the online server at the National Center for Biotechnology Information (http://blast.ncbi.nlm.nih.gov/Blast).

The unknown sequences were aligned with known published sequences of the major genotypes using the alignment program MULTALIN (http://multalin. toulouse.inra.fr/multalin) [22]. The genotypes of samples were determined based on this comparison.

The phylogenetic tree was constructed by the neighbor-joining method of genetic distance calculated by the MEGA 6 (http://www.megasoftware.net) [23].

Genotype identification was carried out using a real-time FRET PCR assay based on sequence analysis of the 18S rRNA gene, and sensitivity and specificity were evaluated in comparison with traditional parasitological techniques.

\section{Clinical course}

Observing the clinical course, we collected data on (1) time between onset of symptoms and diagnosis of $\mathrm{AK}$, (2) clinical signs of AK, (3) coinfection with bacteria or fungi, (4) conservative treatment, (5) surgical treatment, and (6) outcome. For the outcome, we defined "success" if no sign of active AK was observed at least for 6 months, without the use of topical antiamebic therapy. We defined "failure" if AK persisted or recurred.

\section{Results}

\section{Clinical data}

Seven patients with AK had the diagnosis 1.36 months after first symptoms, at the Department of Ophthalmology of Semmelweis University as shown in Figure 1.

At the first presentation, pseudendritiformic epithelopathy/dirty epithelium (four eyes, 57.1\%), multifocal stromal infiltrates (five eyes, 71.4\%), ring infiltrate (three eyes, $42.8 \%$ ), and perineuritis (one eye, 14.3\%) were observed. The clinical data are summarized in Table II. 

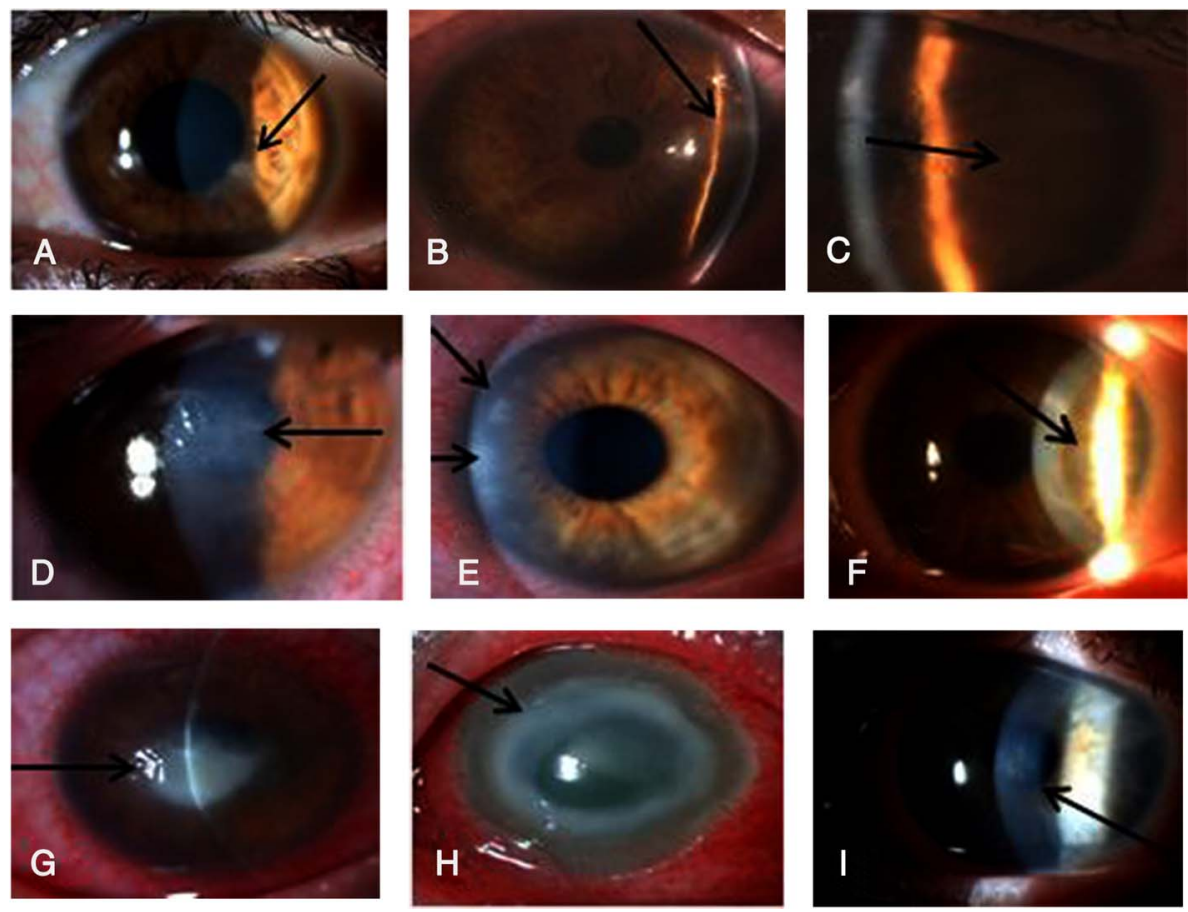

Figure 1. Clinical images of Acanthamoeba keratitis in patients 1-7. Patient 1: multifocalstromal infiltrates. B and C: Patient 2: perineuritis (B) (arrow) and pseudodendritiformic epitheliopathy (“dirty epithelium") (C). D: Patient 3: multifocal stromal infiltrates and incomplete ring infiltrate (arrow). E and F: Patient 4: multifocal stromal infiltrates (arrows) (E) and ring infiltrate (F) (arrow). G: Patient 5: deep stromal infiltrate (arrow) and fine slightly visible multifocal stromal infiltrates. H: Patient 6: epithelial erosion and broad ring infiltrate (arrow). I: Patient 7: multifocal stromal infiltrates (arrow)

AK was healed without later recurrence in two eyes (28.5\%) using tripletopical therapy (TTT; polyhexamethylene biguanide, propamidine isethionate, and antibiotics/neomycin) in three eyes (42.8\%) following additional penetrating keratoplasty.

In Patient 3, AK recurred following successful application of TTT and was treated successfully with repeated TTT. In Patient 7, no follow-up data were available, following diagnosis. We could not observe similarities in genotype and clinical course or the necessity of corneal transplantation.

\section{Cultivation}

All investigated samples revealed Acanthamoeba that were able to grow at $36{ }^{\circ} \mathrm{C}$, the approximate temperature of the human host. Microscopical cultivation 


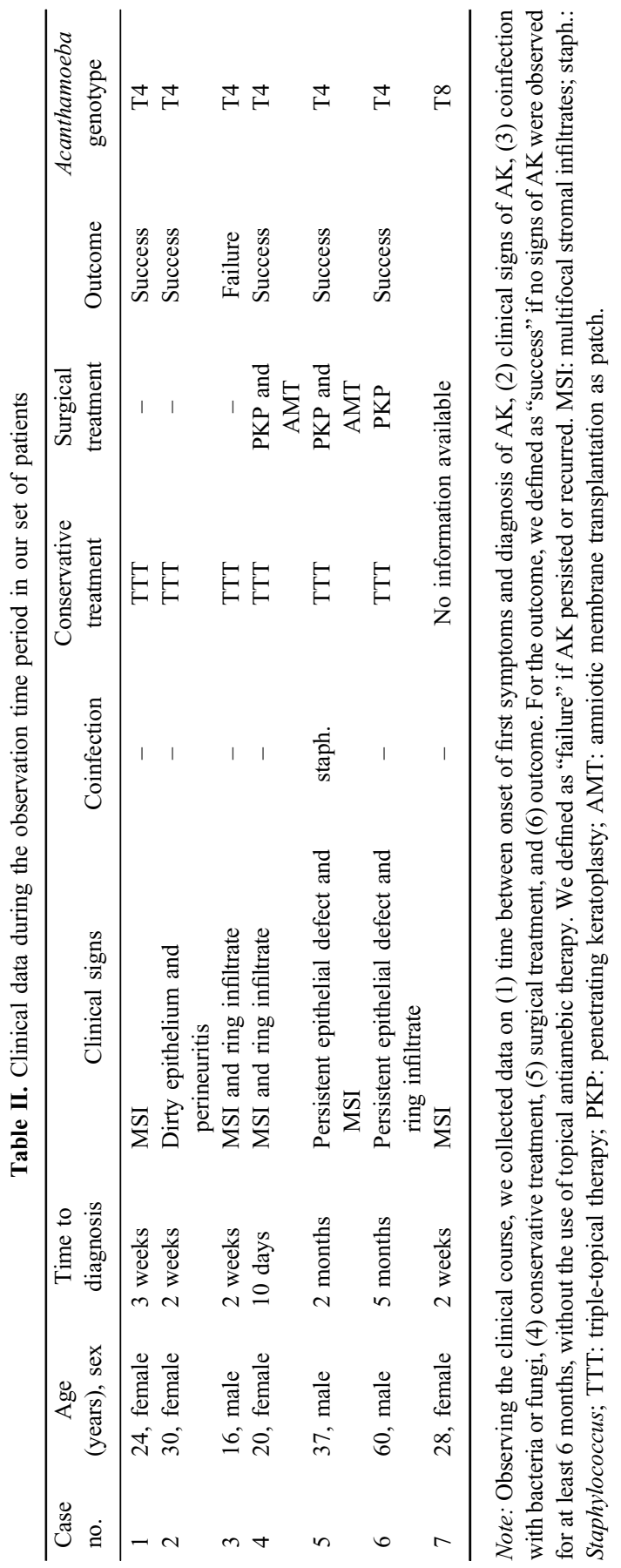


was successful in six samples. Probably due to low quantity of corneal scraping in the seventh sample, one sample showed negative result for cultivation. Further examination of the obtained results was carried out by FRET PCR.

\section{Molecular methods}

This study reports successful PCR amplification for seven (four females and three males) positive cases. The samples for Acanthamoeba-positive patients, detected by PCR method, were sequenced to identify the species (NCBI Bank: Patient_1-KF873021_T4, Patient_2-KP337296_T4, Patient_3-KU356846_T4, Patient_4-KU356848_T4, Patient_5-KR494236_T4, Patient_6-KJ094693_T4, and Patient_8-MF065931_T8). Sequence analysis using a BLAST search indicated an identity of $>98 \%$ with Acanthamoeba $18 \mathrm{~S}$ rRNA gene reference sequences. It was found that the obtained sequences of amebae isolates from the cases belonged to the T4 and T8 genotypes Acanthamoeba spp. neighbor-joining analysis inferred relationships between the PCR products isolated from corneal scrapings and reference strains obtained from NCBI GenBank are shown in Figure 2, respectively.

\section{Discussion and Conclusions}

Detection of Acanthamoeba can be rapidly achieved using real-time FRET molecular methods. For diagnostic purposes, the detection of Acanthamoeba at the genus level is sufficient to recognize whether an individual is infected or not $[24,25]$.

Literature describes T4 genotype Acanthamoeba, as the most common in the environment; however, in AK, prevalence of T4 genotype is even more common. The molecular analysis conducted in this study confirmed, in our series of patients, the T4 genotype to be the most frequent cause of AK. In addition, one isolate was T8 genotype and was first associated with AK [26]. These results are consistent with previous findings indicating that $\mathrm{T} 4$ is worldwide predominant in $\mathrm{AK}$ $[27,28]$.

Nevertheless, a heterogeneous virulence of different Acanthamoeba strains has been observed in our case series, for different Acanthamoeba strains. In our opinion, different reference sequences of T4 or other Acanthamoeba genotypes may also be important in prognosting disease progression.

For an appropriate diagnosis, ophthalmological signs of AK must be known. These are "dirty epithelium" (pseudodendritiformic epitheliopathy), non-healing epithelial defects or ulcer, mono- or multifocal stromal infiltrates, perineuritis, ring 


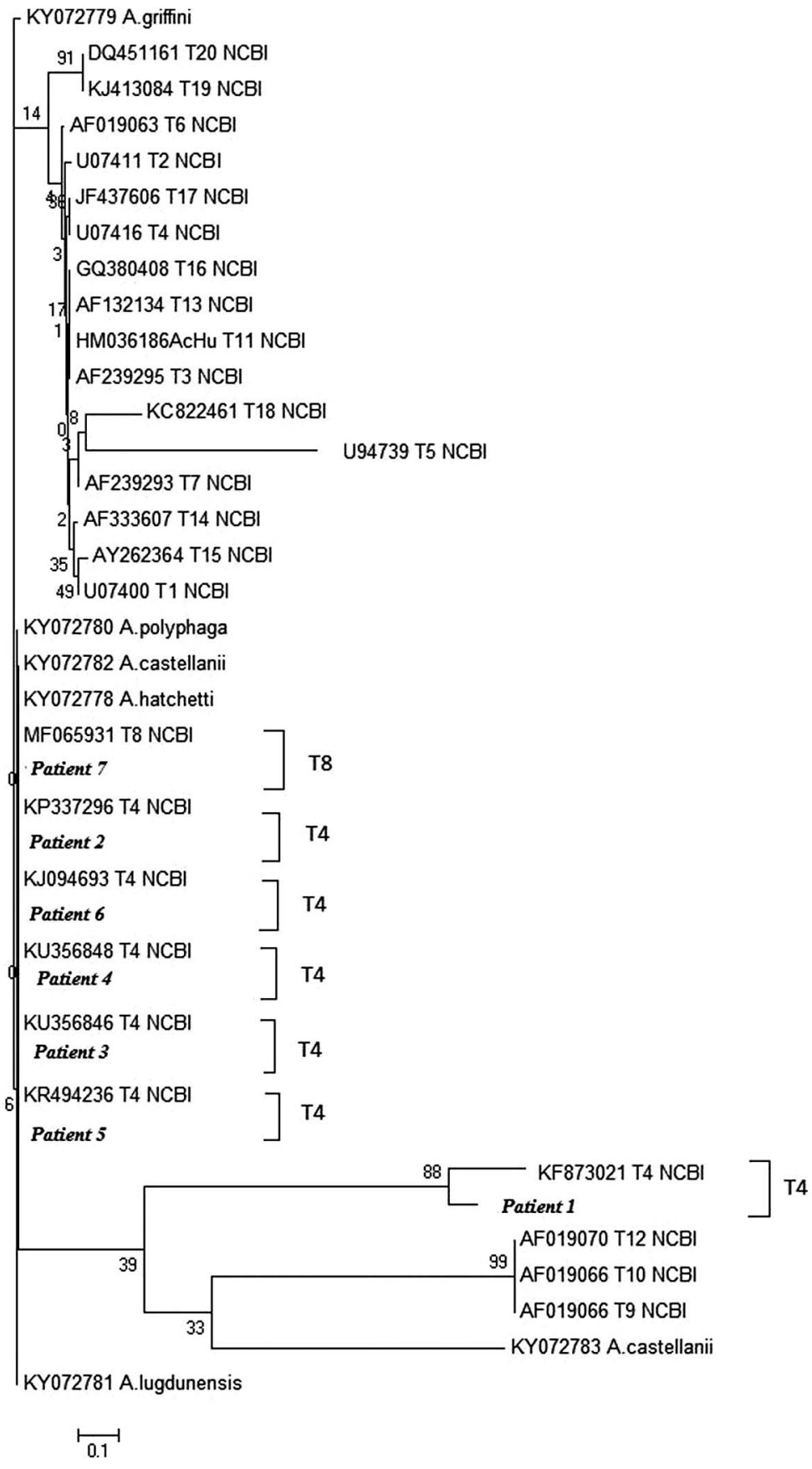

Figure 2. Phylogenetic relations of Acanthamoeba species PCR product Patient_1, Patient_2, Patient_3, Patient_4, Patient_5, Patient_6, Patient_7 and reference strains from NCBI GenBank inferred by neighbor-joining analysis from pairwise comparisons (180-bp fragments) 
infiltrate, and in later stages anterior syneciae, iris atrophy, secondary glaucoma, mature cataract, scleritis and chorioretinitis, or even blindness. To date, about $75 \%$ of ophthalmologists miss the appropriate clinical diagnosis in AK. Faulty typical diagnosis is herpetic keratitis in most of the cases; however, bacterial or mycotic keratitis may also be suggested [29-33]. However, cooperation between clinicians and experts in laboratory diagnostics is indispensable, for adquate treatment in time of these patients.

Nowadays, there is no standardized treatment of $\mathrm{AK}$, as no previous randomized controlled studies have been performed in this rare and often heterogeneous corneal disease. In addition, Acanthamoeba cysts are often resistant or become resistant during treatment to all available topical therapeutic options. The therapeutic regimen used in the recent times includes biguanides (pethylene biguanide $0.02 \%$ and chlorhexidine $0.02 \%$ ), diamidine (propamidine isethionate $0.1 \%$ and hexamidine $0.1 \%$ ), and antibiotics/neomycin as TTT. Antimycotic eye drops, propidium iodide, and miltefosine may also be effective against Acanthamoeba strains. Besides conservative treatment, in advanced cases or in cases without response to topical therapy, penetrating keratoplasty, amniotic membrane transplantation, or crosslinking treatment may be performed [34].

To the best of our knowledge, this is the first study to analyze a correlation between Acanthamoeba genotype and clinical course. However, we could not determine a relationship between both of them in these series of patients. To date, there is no standard treatment of AK; however, we also did not find a study analyzing relationship between genotype and Acanthamoeba susceptibility to different agents. In our opinion, this also needs development in the following years.

There are only case series on safety and effectivity of medical and surgical treatment of $\mathrm{AK}$ and to date there are no randomized controlled clinical studies. In Hungary, we suggest topical application of polyhexamethylene biguanide, propamidine isethionate, and antibiotics/neomycin as TTT in case of AK [35].

During the first 2 days, a "surprise attack" or "flash war" is initiated with polyhexamethylene biguanide and propamidine isethionate every quarter to half an hour day and night. Then, until the sixth day, polyhexamethylene biguanide and propamidine isethionate are applied every hour and only over the day. Following 4 weeks, eyedrop use is reduced to every $2 \mathrm{~h}$. In addition, antibiotics/neomycin $5 \times$ a day is also applied for some week.

Worldwide incidence of AK increases, presumably due to the increasing use of CLs [36, 37]. To the best of our actual knowledge, combination therapy using diamidine, biguanide, and antibiotics should be continued in descending dosis until 1 year.

In Hungary, AK has been developed through Acanthamoeba genotypes T4 and T8 in the past 3 years. Analyzing seven patients, we could not determine a 
relationship between Acanthamoeba genotype and clinical course of the disease. We suggest the development of an international database on AK causative isolates for better understanding of the disease course and better treatment of these patients.

\section{Conflict of Interest}

The authors declare no conflict of interest. EO assures that there are no links with a company whose product is mentioned in the article or a company that distributes a competing product. The authors also state that the presentation of the topic is independent and the presentation of the content is product-neutral.

\section{References}

1. Shatilovich, A., Shmakova, L., Gubin, S., Goodkov, A., Gilichinsky, D.: Viable protozoa in late Pleistocene and Holocene permafrost sediments. Dokl Biol Sci 401, 136-138 (2005).

2. Podlipaeva, I., Shmakov, L. A., Gilichinski, D. A., Gudkov, A. V.: Heat shock protein of hsp70 family revealed in some contemporary freshwater amoebids and in Acanthamoeba sp. excysted from cysts isolated from permafrost samples. Tsitologiia 48, 691-694 (2006).

3. Nuprasert, W., Putaporntip, C., Pariyakanok, L., Jongwutiwes, S.: Identification of a novel t17 genotype of Acanthamoeba from environmental isolates and t10 genotype causing keratitis in Thailand. J Clin Microbiol 48, 4636-4640 (2010).

4. Jones, D., Visvesvara, G., Robinson, N.: Acanthamoeba polyphaga keratitis and Acanthamoeba uveitis associated with fatal meningoencephalitis. Trans Ophthalmol Soc UK 95, 221-231 (1975).

5. Siddiqui, R., Khan, N. A.: Biology and pathogenesis of Acanthamoeba. Parasit Vectors 5, 6 (2012).

6. Page, F. C.: Taxonomic criteria forlimax amoebae, with descriptions of 3 new species of Hartmannella and 3 of Vahlkampfia. J Protozool 14, 499-521 (1967).

7. Corsaro, D., Walochnik, J., Köhsler, M., Rott, M. B.: Acanthamoeba misidentification and multiple labels: Redefining genotypes T16, T19, and T20 and proposal for Acanthamoeba micheli sp. nov. (genotype T19). Parasitol Res 114, 2481-2490 (2015).

8. Schroeder, J. M., Booton, G. C., Hay, J., Niszl, I. A., Seal, D. V., Markus, M. B., Fuerst, P. A., Byers, T. J.: Use of subgenic $18 \mathrm{~S}$ ribosomal DNA PCR and sequencing for genus and genotype identification of Acanthamoebae from humans with keratitis and from sewage sludge. J Clin Microbiol 39, 1903-1911 (2001).

9. Risler, A., Coupat-Goutaland, B., Pélandakis, M.: Genotyping and phylogenetic analysis of Acanthamoeba isolates associated with keratitis. Parasitol Res 112, 3807-3816 (2013).

10. Orosz, E., Farkas, Á., Kucsera, I.: Laboratory diagnosis of Acanthamoeba keratitis in Hungary. Acta Microbiol Immunol Hung 63, 293-299 (2016).

11. Khan, N. A., Jarroll, E. L., Paget, T. A.: Molecular and physiological differentiation between pathogenic and nonpathogenic Acanthamoeba. Curr Microbiol 45, 197-202 (2002). 
12. Maghsood, A. H., Sissons, J., Rezaian, M., Nolder, D., Warhurst, D., Khan, N. A.: Acanthamoeba genotype T4 from the UK and Iran and isolation of the T2 genotype from clinical isolates. J Med Microbiol 54, 755-759 (2005).

13. Di Cave, D., Monno, R., Bottalico, P., Guerriero, S., D’Amelio, S., D’Orazi, C., Berrilli, F.: Acanthamoeba T4 and T15 genotypes associated with keratitis infections in Italy. Eur J Clin Microbiol Infect Dis 28, 607-612 (2009).

14. Iovieno, A., Oechsler, R. A., Ledee, D. R., Miller, D., Alfonso, E. C.: Drug-resistant severe Acanthamoeba keratitis caused by rare T5 Acanthamoeba genotype. Eye Contact Lens 36, 183-184 (2010).

15. Arnalich-Montiel, F., Lumbreras-Fernández, B., Martín-Navarro, C. M., Valladares, B., Lopez-Velez, R., Morcillo-Laiz, R., Lorenzo-Morale, J.: Influence of Acanthamoeba genotype on clinical course and outcomes for patients with Acanthamoeba keratitis in Spain. J Clin Microbiol 52, 1213-1216 (2014).

16. Walochnik, J., Scheikl, U., Haller-Schober, E. M.: Twenty years of Acanthamoeba diagnostics in Austria. J Eukaryot Microbiol 62, 3-11 (2015).

17. Omaña-Molina, M., Vanzzini-Zago, V., Hernandez-Martinez, D., Gonzalez-Robles, A., Salazar-Villatoro, L., Ramirez-Flores, E., Oregon-Miranda, E., Lorenzo-Morales, J., Martinez-Palomo, A.: Acanthamoeba genotypes T3 and T4 as causative agents of amoebic keratitis in Mexico. Parasitol Res 115, 873-878 (2016).

18. Martín-Pérez, T., Criado-Fornelio, A., Martínez, J., Blanco, M. A., Fuentes, I., Pérez-Serrano, J.: Isolation and molecular characterization of Acanthamoeba from patients with keratitis in Spain. Eur J Protistol 61, 244-252 (2017).

19. Page, F. C.: A New Key to Freshwater and Soil Gymnamoebae. Freshwater Biological Association, Ambleside, UK, 1988, 122 p.

20. Orosz, E., Farkas, Á., Ködöböcz, L., Becsák, P., Danka, J., Kucsera, I., Füleky, G.: Isolation of Acanthamoeba from the rhizosphere of maize and lucerne plants. Acta Microbiol Immunol Hung 60, 29-39 (2013).

21. Altschul, S. F., Gish, W., Miller, W., Myers, E. W., Lipman, D. J.: Basic local alignment search tool. J Mol Biol 215, 403-410 (1990).

22. Corpet, F.: Multiple sequence alignment with hierarchical clustering. Nucleic Acids Res 16, 10881-10890 (1988).

23. Tamura, K., Stecher, G., Peterson, D., Filipski, A., Kumar, S.: MEGA6: Molecular evolutionary genetics analysis version 6.0. Mol Biol Evol 30, 2725-2729 (2013).

24. Rivera, W. L., Edric, D. V.: $18 \mathrm{~S}$ ribosomal DNA genotypes of Acanthamoeba species isolated from contact lens cases in the Philippines. Parasitol Res 105, 1119-1124 (2009).

25. Alves, D. S., Moraes, A. S., Nitz, N., de Oliveira, M. G., Hecht, M. M., Gurgel-Gonçalves, R., Cuba, C. A.: Occurrence and characterization of Acanthamoeba similar to genotypes T4, T5, and T2/T6 isolated from environmental sources in Brasília. Exp Parasitol 131, 239-44 (2012).

26. Orosz, E., Szentmáry, N., Kiss, H. J., Farkas, Á., Kucsera, I., Nagy, Z. Z.: First report of Acanthamoeba genotype T8 human keratitis. Acta Microbiol Immunol Hung 65, 73-79 (2018).

27. Visvesvara, G. S., Hercules, M., Schuster, F. L.: Pathogenic and opportunistic free-living amoebae: Acanthamoeba spp., Balamuthia mandrillaris, Naegleria fowleri and Sappinia diploidea. FEMS Immunol Med Microbiol 50, 1-26 (2007). 
28. Maciver, S. K., Asif, M., Simmen, M. W., Lorenzo-Morales, J.: A systematic analysis of Acanthamoeba genotype frequency correlated with source and pathogenicity: T4 is confirmed as a pathogen-rich genotype. Eur J Protistol 49, 217-221 (2013).

29. Szentmáry, N., Goebels, S., Matoula, P., Schirra, F., Seitz, B.: Die Akanthamöbenkeratitis einseltenes und oft spät diagnostiziertes Chamäleon [Acanthamoeba keratitis - A rare and often late diagnosed disease]. Klin Monbl Augenheilkd 229, 521-528 (2012).

30. Szentmáry, N., Daas, L., Matoula, P., Goebels, S., Seitz, B.: Akanthamöbenkeratitis. Ophthalmologe 110, 1203-1210 (2013).

31. Szentmáry, N., Seitz, B., Nagy, Z. Z.: Acanthamoeba keratitis - Clinical presentation, diagnosis and treatment. Szemészet 151, 23-28 (2014).

32. Daas, L., Szentmáry, N., Eppig, T., Langenbucher, A., Hasenfus, A., Roth, M., Saeger, M., Nölle, B., Lippmann, B., Böhringer, D., Reinhard, T., Kelbsch, C., Messmer, E., Pleyer, U., Roters, S., Zhivov, A., Engelmann, K., Schrecker, J., Zumhagen, L., Thieme, H., Darawsha, R., Meyer-ter-Vehn, T., Dick, B., Görsch, I., Hermel, M., Kohlhaas, M., Seitz, B.: Das Deutsche Akanthamöbenkeratitis-Register - Erste Ergebnisse einer multizentrischen Erhebung [The German Acanthamoeba keratitis register: Initial results of a multicenter study]. Ophthalmologe 112, 752-763 (2015).

33. Lee, M. H., Abell, R. G., Mitra, B., Ferdinands, M., Vajpayee, R. B.: Risk factors, demographics and clinical profile of Acanthamoeba keratitis in Melbourne: An 18-year retrospective study. Br J Ophthalmol 102, 687-691 (2018).

34. Szentmáry, N., Daas, L., Shi, L., Laurik, K. L., Seitz, B.: SOP Akanthamöbenkeratitis Klinische Zeichen, Diagnose, Therapie [SOP Acanthamoeba keratitis - Clinical signs, diagnosis, therapy]. Augeheilkunde Up2date 7, 281-287 (2017).

35. Gyenes, A., Orosz, E., Sándor, G. L., Fries, F. N., Seitz, B., Nagy, Z. Z., Szentmáry, N.: Early diagnosis and successful medical treatment of Acanthamoeba keratitis. Klin Monbl Augenheilkd 235, 1407-1410 (2018).

36. Thebpatiphat, N., Hammersmith, K. M., Rocha, F. N., Rapuano, C. J., Ayres, B. D., Laibson, P. R., Eagle, R. C. Jr., Cohen, E. J.: Acanthamoeba keratitis: A parasite on the rise. Cornea 26, 701-706 (2007).

37. Lee, W. B., Gotay, A.: Bilateral Acanthamoeba keratitis in Synergeyes contact lens wear: Clinical and confocal microscopy findings. Eye Contact Lens 36, 164-169 (2010). 